\title{
Leave no Tooth Behind - A Retreatment Case Report
}

\section{Reaba Rebecca Thomas ${ }^{1 *}$, Aby John ${ }^{1}$, Shashi Rashmi Acharya ${ }^{2}$ and Archit Kedia ${ }^{3}$}

${ }^{1}$ Private Practitioner, Department of Conservative Dentistry and Endodontics, Dubai, United Arab Emirates

${ }^{2}$ Professor, Department of Conservative Dentistry and Endodontics, MCODS, Manipal, India

${ }^{3}$ Private Practitioner, Avant Dental, Salt Lake, Kolkatal, India

*Corresponding Author: Reaba Rebecca Thomas, Private Practitioner, Department of Conservative Dentistry and Endodontics, Dubai, United Arab Emirates.
Received: December 26, 2020

Published: January 23, 2021

(C) All rights are reserved by Reaba Rebecca Thomas.

\begin{abstract}
In a dental practice, it is becoming increasingly common to have endodontic cases which are seen to fail. The spike in the number of endodontic treatments carried out has consequently led to the increase in the number of failures seen and complications in their management. Endodontic retreatment is favored over dental extraction due to the desire to preserve the tooth and prevent adverse consequences in terms of functional and psychological effects that the loss of a tooth would have on a patient. This article showcases a clinical case that required endodontic retreatment to combat any complications that could arise and lead ultimately to the extraction of the tooth. Considering that the tooth in question has a special importance with a physiognomic role, we resorted to its endodontic retreatment and we have covered it with a porcelain fused to metal crown.
\end{abstract}

Keywords: Endodontic Failure; Retreatment; Incomplete Obturation; Crown

\section{Introduction}

In a dental practice, it is becoming increasingly common to have endodontic cases which are seen to fail. The spike in the number of endodontic treatments carried out has consequently led to the increase in the number of failures seen and complications in their management. Endodontic retreatment is favored over dental extraction due to the desire to preserve the tooth and prevent adverse consequences in terms of functional and psychological effects that the loss of a tooth would have on a patient. The primary goal of nonsurgical endodontic retreatment is to establish a healthy periodontium by ensuring complete removal of the initial filling materials, disinfection, and re-obturation of the root canal system $[1,2]$. There has been a steady increase in the number of cases which is truly staggering, and can be described as the good-news-bad-news dilemma. The idealist amongst us would like to believe that today millions of teeth are being salvaged through endodontics but the realist however knows that today thousands of teeth are endodontically failing every year for a variety of reasons.
The rationale for retreatment is that regardless of the aetiology, all endodontic failures can be ultimately attributed to leakage and/or bacterial contamination. Routinely, healing of lesions of endodontic origin are seen post extraction due to the complete elimination of the contents within the root canal system. Endodontic retreatment can also approach $100 \%$ success rate if the entire contents of the root canal system are eliminated and an adequate seal is provided.

The many reasons to attempt a retreatment are perforations, broken instruments, coronal leakage, post related errors, radicular fractures, missed canals, improper obturation and operator limitations. The main objective of endodontic retreatment is cleansing the canals of irritants, mostly microorganisms, that had survived previous treatment or had infiltrated the canals post treatment. Since this involves the repetition of the steps involved in a root canal therapy, and is also consistent with the biological rationale, retreatments are advocated whenever possible. 
This case report showcases the need for retreatment due to coronal leakage of the restoration, post related errors and improper obturation.

\section{Case Report}

A female patient of 32 years of age presented with the chief complaint of pain for 2 months in the lower left back tooth region. The endodontic treatment for the tooth in question had been completed 2 years ago. She also gave a history of occasional pus discharge from the same tooth ever since the completion of the endodontic treatment. The patient's other demographic details like medical history, family history, general examination was either normal or non-contributory.

Oral examination revealed composite restoration with evidence of secondary caries with respect to \#36. There was presence of a $10 \mathrm{~mm}$ periodontal pocket on the buccal aspect with pus discharge from the gingival sulcus. The buccal aspect of the tooth showed evidence of a draining sinus (Figure 1).

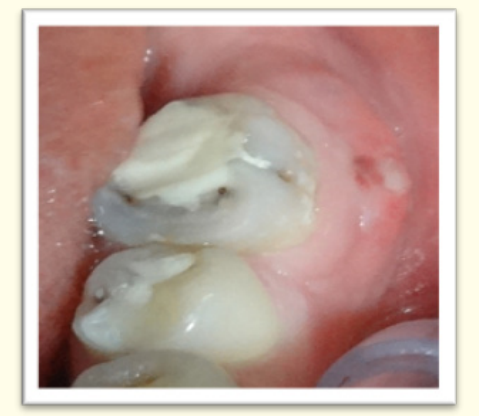

Figure 1: Intraoral view of tooth with draining sinus.

Radiographic examination showed insufficiently obturated root canals with a radio-opaque object seen projecting from the distal canal suggestive of a metal post. Periodontal widening was observed with radiolucency present in the furcation area (Figure 2).

A diagnosis of acute apical periodontitis secondary to incomplete root canal treatment \#36 was made and retreatment was suggested with respect to the same.

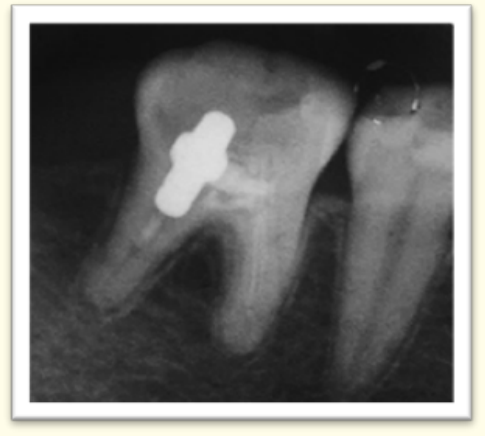

Figure 2: Pre-operative Radiograph.

Composite restoration in \# 36 was removed and secondary caries was excavated. The gutta-percha(GP) in the pulp chamber and the metal post in the distal canal were evidently visible. The metal post was removed with the help of ultrasonics and artery forceps (Figure 3,4). Pre-endodontic composite build-up was the done and the tooth was isolated using a rubber dam. Canals were located after GP removal and the orifices enlarged using the Protaper Sx Rotary file. The canals were negotiated and working length was established (Figure 5). Canals were prepared using Mtwo ${ }^{\circ}$ retreatment file system and irrigated with 3\% sodium hypochlorite and activated using EndoActivator. The canals were then obturated and composite final restoration was placed. In order to restore the integrity of the mandibular arch, a porcelain fused to metal crown was delivered as the final coronal restoration. The first mandibular, being an important tooth, was saved by a timely intervention and carrying out the retreatment allowed for the remission of clinical symptoms and prevented any periodontal complications from arising.

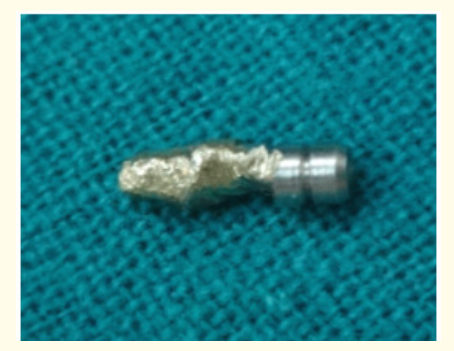

Figure 3: The Retrieved Post 


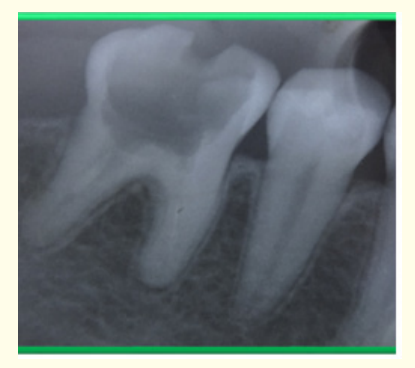

Figure 4: Removal of Gutta Percha.

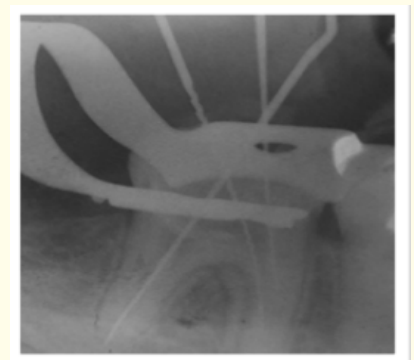

Figure 5: Working Length Determination.

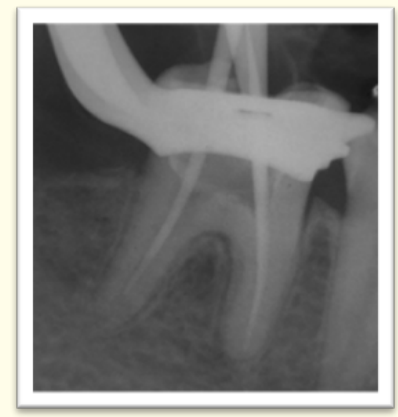

Figure 6: Master Cone Placement.

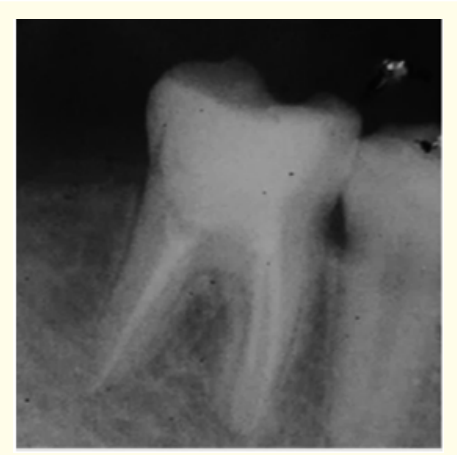

Figure 7: Obturation and Composite Core Buildup.

\section{Discussion}

The failure of an initial endodontic treatment can be solved one of three ways.The first and most indicated solution is represented by endodontic retreatment. Another option is performing an apicoectomy along with retrograde sealing of the root canals and the final option, which is the least desirable, is extraction of the tooth. The latter is necessary if the first two options cannot be achieved. When access to the root canals can be easily attained then it is preferable to go with the first option and perform a non-surgical retreatment procedure. The goal of a retreatment is the complete removal of all root canal contents, followed by proper cleaning, shaping and three-dimensional filling of the previously obturated canals with an inert material like gutta percha.

Some studies have given evidence that $45 \%$ of endodontically treated teeth showed the presence of persistent apical periodontitis $[3,4]$. This was seen to be due to microorganisms that had survived the endodontic treatment. However this was easily resolved by an orthograde endodontic retreatment [5]. During retreatment, it is imperative to remove the root filling material completely, so as to eliminate all residual microorganisms and create conditions favorable for periradicular healing [6].

The success rate of endodontic retreatments have been analysed in many studies, based on the presence or absence of periapical lesions. Holland and colleagues have analyzed seventeen such studies [7]. It has been concluded that the success rate is higher in the absence of a periradicular lesion and the rate varies from a minimum of $8 \%$ to a maximum of $85 \%$ when a lesion is present. In cases where infection is not present and retreatment is carried out by a skilled clinician sucess rates are $100 \%$ as shown by Friedman., et al. [8] Also Sjogren and colleagues [9], obtained a 98\% success rate while performing endodontic retreatments on 173 roots in the absence of periapical lesions. The success rate can be estimated at not more than $70 \%$,if prior to the commencement of endodontic retreatment a periapical lesion is present [8].

The decision to retain or remove a post is based on multiple factors like operator judgement, training and experience of the clinician, knowledge of the tooth anatomy and the location of the tooth in the oral cavity.

There is a myriad of techniques to remove a post such as use of transmetal burs, ultrasonics, various mechanical post removal systems, rotosonic vibration burs or a combination of all the aforemen- 
tioned. The various factors influencing the removal of Gutta Percha are the curvature and configuration of the canal, cross-sectional dimension of the tooth, total length of the canal and the technique of obturation done. A combination of methods is generally required to provide safe, efficient and complete elimination of gutta-percha and sealer from the root canal systems. Use of retreatment rotary files, hand files ultrasonic instruments, instruments with heat or chemical and chemical impregnated paper points are some of the commonly used techniques for the removal of Gutta-percha during a retreatment.

Mtwo $^{\circ}$ retreatment file system is designed specifically for the removal of gutta-percha. It possesses a cutting tip with constant helical angle which allows the easy progression of the file into the filling, without exerting pressure.

There has been more advancement in clinical endodontics in the past 20 years than, perhaps the previous 100 years combined. With the advent of four game changing technologies, the face of endodontic retreatment has been completely transformed.

The advent of loupes and microscopes allowed superior vision to become attainable thereby drastically improving success rates. Ultrasonic instruments have made canal preparations minimally invasive, efficient and precise. Rotary instruments have made canal preparations more predictable and successful. The emergence of restorative materials like Mineral Trioxide Aggregate (MTA), countless teeth previously considered hopeless, can now be salvaged. All these innovations have had a large role to play in making endodontic retreatment a feasible and reliable science.

\section{Conclusion}

In the years to come endodontic treatments and retreatments will continue to become more common. The foundation of restorative and reconstructive dentistry however will continue to be properly performed endodontic treatments. With the growing appreciation for the importance of the attachment apparatus, a naturally retained root will soon come to be accepted as the ultimate implant.

\section{Bibliography}

1. Nair PN. "On the causes of persistent apical periodontitis: a review". International Endodontic Journal 39 (2006): 249-281.
2. Ricucci D and Siqueira JF Jr. "Biofilms and apical periodontitis: study of prevalence and association with clinical and histopathologic findings". Journal of Endodontics 36 (2010): 12771288.

3. Kirkevang Horsted-Bindslev P and Orstavik D Wenzel. "Frequency and distribution of endodontically treated teeth and apical periodontitis in an urban Danish population". International Endodontic Journal 34 (2001): 198-205.

4. Dugas NN., et al. "Periapical health and treatment quality assessment of root-filled teeth in two Canadian populations". International Endodontic Journal 36 (2003): 181-192.

5. Friedman S. "Considerations and concepts of case selection in the management of post-treatment endododntic disease". Endodontics Topics 1 (2002): 45-64.

6. Stabholz A and Friedman S. "Endodontic retretment - case selection and technique. Part 2: treatment planning for retreatment". Journal of Endodontics 14 (1988): 607-614.

7. Holland R., et al. "Influence of bony resorption on endodontic treatment". Oral Surgery, Oral Medicine, Oral Pathology 55 (1983): 191.

8. Friedman S. "Treatment outcome and prognosis of endodontic therapy". In: Ørstavik D, Pitt Ford TR, editors. Essential endodontology. London: Blackwell Scientific (1998): 367-401

9. Sjögren U., et al. "Factors affecting the long-term results of endodontic treatment". Journal of Endodontics 16 (1990): 498.

\section{Assets from publication with us}

- Prompt Acknowledgement after receiving the article

- Thorough Double blinded peer review

- Rapid Publication

- Issue of Publication Certificate

- High visibility of your Published work

Website: www.actascientific.com/

Submit Article: www.actascientific.com/submission.php

Email us: editor@actascientific.com

Contact us: +919182824667 